\title{
La pertinencia de la educación desde la perspectiva de los estudiantes en una universidad pública mexicana
}

\section{The pertinence of education from the students' perception in a Mexican State university}

\begin{abstract}
Juan Manuel Hernández
Doctor en Estudios Sociales, Universidad Autónoma Metropolitana, México. Profesorinvestigador, Departamento de Sociología, Universidad Autónoma Metropolitana Iztapalapa, México. Especialista en indicadores sobre resultados de la educación, reforma universitaria y mercados de trabajo. Coordinador del Programa de Maestría y Doctorado en Estudios Sociales, línea Estudios Laborales, Universidad Autónoma Metropolitana Iztapalapa. Investigador Nivel I - Sistema Nacional de Investigadores (SNI).
\end{abstract}

Javier Rodríguez

Doctor en Sociología por la Universidad Nacional Autónoma de México. Profesor investigador, Departamento de Sociología, Universidad Autónoma Metropolitana Iztapalapa, México. Especialista en estudios sobre deserción y permanencia escolar en el nivel terciario de la educación, reforma universitaria y mercados de trabajo. Coordinador del Laboratorio de Docencia y del Estudiante Universitario, Departamento de Sociología, Universidad Autónoma Metropolitana - Iztapalapa. Investigador Nivel I - Sistema Nacional de Investigadores (SNI).

Fecha de recibido: $24 / 03 / 2015$

Fecha de aceptado: 16/04/2015

\section{Resumen}

Esta investigación muestra cómo el alumnado percibe la actividad de la universidad en cuanto a la pertinencia de la formación que recibe. La pertinencia es analizada con base en tres dimensiones centrales de los aprendizajes que, se supone, los alumnos adquieren en su paso por el campus: aprendizajes para hacer, para convivir y para seguir aprendiendo a lo largo de la vida.

La información analizada proviene de la encuesta "La reforma universitaria desde la perspectiva de los alumnos, UAMI 20141" que fuera realizada sobre una muestra de 283 estudiantes, representativa de la población estudiantil con actividad regular en la Universidad Autónoma Metropolitana, campus Iztapalapa (UAMI). Este campus se ubica en la zona oriente de la ciudad de México. El análisis de las percepciones estudiantiles, relacionadas con cada una de las dimensiones mencionadas, se basó en la construcción de tres índices sintéticos correspondientes.

Los resultados indicaron que los estudiantes tienen la expectativa de que la formación recibida debería tener utilidad, sobre todo, para "aprender a hacer" "y "aprender a aprender", y no tanto para "aprender a convivir". Sin embargo, la labor educativa concreta de la universidad, de acuerdo con la propia valoración estudiantil, guarda cierta relación con las expectativas de "aprender a hacer" y "aprender a convivir", más no tanto con las de "aprender a aprender".

Palabras clave: pertinencia, relevancia, educación superior, percepción de los estudiantes. 


\begin{abstract}
This research shows how pertinent students perceive their University training is. This pertinence is analyzed on three central dimensions of learning that, supposedly, students acquire during their academic training, such dimensions being: learning to do, learning to live together and continuous learning throughout life.

Analyzed data are obtained from the survey 'The university reform from the perspective of students, UAMI 2014', which was applied on a sample of 283 students. This is a representative sample of the student population with regular activity at the Universidad Autónoma Metropolitana campus Iztapalapa (UAMI), which is located on the eastern area of Mexico City. The analysis of the students' perceptions referred to each of the three dimensions above mentioned is based on the design of three synthetic indexes.

Results reveal that students expect the academic training and knowledge to be useful especially for "learning to do" and "learning to learn", but not as much as "learning to live together". However, according to the students' perceptions, the concrete educational work of the university is more consistent with their expectations of "learning to do" and "learning to live together", than that of "learning to learn".
\end{abstract}

Keywords: pertinence, relevance, higher education, students' perceptions.

\title{
Introducción
}

La Universidad Autónoma Metropolitana (UAM) fue fundada durante el ciclo de reforma que alcanzó su clímax en la década de 1970 en cuanto a la expansión de la educación superior pública mexicana. Esta institución nació en 1974. De los tres campus que inicialmente conformaron la institución, el de Iztapalapa, en la zona oriente de la Ciudad de México, fue el primero en abrir sus puertas, el 30 de septiembre. La UAM actualmente cuenta con cinco campus, cuatro asentados en cada uno de los puntos cardinales de la ciudad de México y el quinto cercano a la ciudad de Toluca, a unos $60 \mathrm{~km}$ al poniente del centro de la ciudad de México. Sus actividades se desarrollan con plan trimestral. En el trimestre 2014-Otoño, la UAM-Iztapalapa (UAMI) concentró casi 30\% de los casi 41500 estudiantes de licenciatura activos en toda la UAM.

La investigación objeto de este reporte permite una aproximación al dato de qué tanto la educación impartida en la UAMI, desde la perspectiva del propio alumnado, guarda una relación con sus expectativas personales sobre el para qué de la formación universitaria. La información analizada fue tomada de la encuesta 'La reforma universitaria desde la perspectiva de los alumnos, UAMI 2014', que contiene otros campos de observación para resolver incógnitas relacionadas con los procesos de cambio que han venido desarrollándose hasta el presente en esta universidad pública. Dicha encuesta fue realizada sobre una muestra de 283 estudiantes, muestra representativa de la población estudiantil con actividad regular.

Este documento comienza dilucidando sobre la connotación que el concepto de pertinencia ha adquirido a partir del uso que le han dado los expertos en calidad educativa. Los autores adhieren a la idea de que hablar de pertinencia educativa es distinto a hablar de relevancia. Coinciden en que la primera tiene que ver con el grado de coherencia entre las realizaciones concretas de los sistemas educativos y las necesidades de los actores individuales, mientras que la segunda alude a la coherencia entre las realizaciones educativas y las necesidades del conjunto social.

Esta investigación muestra cómo el alumnado percibe la actividad de la universidad en cuanto a la pertinencia de la formación recibida. El concepto de pertinencia fue operacionalizado aprovechando parte de lo desarrollado por Delors et al. (1996). Así, la pertinencia es analizada con base en tres dimensiones centrales de los aprendizajes que, se supone, los alumnos adquieren en su paso por el campus: aprendizajes para hacer, 
para convivir y para seguir aprendiendo a lo largo de la vida.

El análisis de las percepciones estudiantiles relacionadas con cada una de las dimensiones mencionadas se basó en la construcción de tres índices sintéticos correspondientes. Los resultados indicaron que los estudiantes tienen la idea de que la formación recibida debería tener utilidad, sobre todo, para "aprender a hacer" y "aprender a aprender", y no tanto para "aprender a convivir". Sin embargo, la labor educativa de la universidad, de acuerdo con la propia valoración estudiantil, está siendo coherente más con sus expectativas de "aprender a hacer" y "aprender a convivir", que con las de "aprender a aprender".

\section{Pertinencia y relevancia de la educación superior}

Dos aspectos de la calidad de la educación superior, difíciles de aprehender, son los relativos a su pertinencia y su relevancia. Diversas conceptualizaciones de la calidad educativa coinciden en que ambas cuestiones se encuentran en el ámbito de los resultados educativos, los cuales implican una relación de correspondencia entre lo que la educación pretende resolver y lo que finalmente llega a alcanzar con su desempeño concreto, pero divergen en si la pertinencia y la relevancia son cuestiones diferentes o si refieren al mismo asunto; si un concepto alude a la solución de necesidades individuales y el otro a la solución de necesidades sociales, o ambos conceptos refieren a ambos tipos de necesidades.

Para Martuscelli y Martínez (2002: 2) el concepto de pertinencia involucra ambos tipos de necesidades. Afirman que "la pertinencia se refiere al grado de correspondencia que debe existir con las necesidades sociales e individuales". La Secretaría de Educación Pública (SEP) coincidió con este planteamiento en su Programa Nacional de Educación 2001-2006 al precisar que la educación mexicana será "pertinente, porque responderá a las necesidades de las personas y a los requerimientos del desarrollo nacional" (SEP, 2001: 72). Para otros estudiosos, como Gago (2002: 10), la pertinencia educativa sólo tiene que ver con la parte social cuando dice que ésta "es el grado en que la educación responde a los problemas, demandas y expectativas de la sociedad". De la Orden (2007: 17) se acerca a Gago cuando, luego de definir la calidad como coherencia interna y externa del sistema, afirma que "la satisfacción de expectativas y necesidades sociales define la calidad de la educación como funcionalidad o pertinencia". La Unesco (1998: 12) concuerda con esta idea al sostener que "la pertinencia de la educación superior debe evaluarse en función de la adecuación entre lo que la sociedad espera de las instituciones y lo que éstas hacen".

Visiones más detalladas, como la del Instituto Nacional para la Evaluación de la Educación, coinciden en que la pertinencia de la educación debe ser separada de la relevancia, ligando la primera a la idea de la correspondencia con las necesidades personales y la segunda a la coherencia con las necesidades de los estudiantes. Asimismo proponen que ambas dimensiones -no sólo la pertinencia- sean consideradas componentes centrales de la calidad educativa. En este sentido, Robles et al. (2012: 22) indican que un sistema educativo de buena calidad tiene entre sus atributos el de ser "relevante porque sostiene una congruencia entre los objetivos de la enseñanza y las necesidades de la sociedad a la que sirve. [Y el de ser] pertinente porque los objetivos, específicamente los del currículo, guardan correspondencia con las necesidades particulares de los alumnos a los que se dirige". Santos y Delgado (2011) sostienen que la atracción y retención de los jóvenes en las escuelas descansa de manera importante "en la pertinencia y relevancia de los contenidos escolares, esto es, en su adecuación a las necesidades individuales y a las de la sociedad", respectivamente. Taccari (2007) afirma que la educación de calidad "supone un entronque con las condiciones particulares de las personas (pertinencia) y con los desafíos de desarrollo que debe afrontar (relevancia)". Martínez et al. (2007: 40) plantean que la pertinencia y la relevancia se verifican cuando los contenidos son acordes con las necesidades educativas de los alumnos y cuando los objetivos son "acordes con las metas sociales". En esta misma línea, Unesco-Orealc (2007: 14) propone un análisis de la relevancia 
y la pertinencia de la educación. La relevancia es tratada en correspondencia con “los cuatro pilares de la educación" -aprender a ser, a conocer, a hacer y a vivir juntos- sugeridos por Delors et al. (1996: 16), los cuales en sí gravitan en la esfera de las necesidades sociales. En cuanto a la pertinencia, el análisis es "organizado a partir de las orientaciones hacia el respeto y valoración de la diversidad, la flexibilidad y adaptabilidad de la oferta educativa a las condiciones particulares de las personas".

Esta investigación adhiere a la idea de que la pertinencia y la relevancia de la educación superior son dos cuestiones diferentes de la calidad educativa, y que la primera tiene que ver con qué tanto los objetivos, acciones y resultados concretos se corresponden, o guardan relación de coherencia, con las necesidades de los alumnos.

No existe un consenso sobre lo que ha de entenderse al hablar de necesidades sociales y necesidades de los alumnos. Sin embargo, ha permeado la idea de que la vinculación de la formación con el sector productivo es una de esas necesidades fundamentales. Desde hace más de medio siglo se han generado propuestas por adecuar la oferta educativa a las necesidades del mercado laboral2. De hecho, los planes nacionales y programas sectoriales alientan la idea de que la educación superior debe encaminarse a ofrecer un capital humano formado para una inserción exitosa en las dinámicas laborales de la globalización y la sociedad del conocimiento.

En la Ley de Coordinación de 1978 (Congreso de la Unión, 1978: 3) apenas se insinuaba -y sólo para las instituciones de educación superior tecnológica-, que debían vincularse con el sector productivo al indicar que habría un órgano consultivo, el "Consejo del Sistema Nacional de Educación Tecnológica" para coordinar sus actividades y "contribuir a vincularlas con las necesidades y el desarrollo del país". Tres años más tarde, el Plan Nacional de la Educación Superior (Anuies, 1981) ya involucraba en esa dinámica a todo el conjunto al plantear que "la educación superior, en los próximos años, deberá incrementar su capacidad de respuesta a los planteamientos que impone el desarrollo, así como su eficacia ante las exigencias de los sectores productivos y los distintos sectores de nuestra sociedad". Estos vestigios de inquietudes en la esfera política por propiciar una mejor coordinación del sistema educativo reverberan con mayor claridad en el actual Plan Nacional de Desarrollo y el Programa Sectorial de Educación 2013-2018. El primero indica expresamente que "resulta prioritario conciliar la oferta educativa con las necesidades sociales y los requerimientos del sector productivo" mediante planes y programas de estudio apropiados que lleven a "lograr una educación de calidad" (Presidencia de la República, 2013: 62). En forma similar, el Programa Sectorial reconoce que "no basta con dar un espacio a los alumnos en las escuelas de todos los niveles; es necesario que la educación forme para [...] la puesta en práctica de habilidades productivas y, en general, para el desarrollo integral de los seres humanos" (SEP, 2013).

Las pretensiones gubernamentales de lograr mayor coordinación entre el sistema educativo y el laboral están ejerciendo presión sobre las instituciones de educación superior. Sin embargo, es necesario considerar que dichos sistemas siguen lógicas y dinámicas no necesariamente coincidentes, por lo cual llegar a una vinculación significativa es una cuestión difícil, y no sólo en México. Dichas presiones, precisamente, han motivado el debate universitario que, para algunos, se ha resuelto en una crítica a la tendencia tanto a "tecnificar" la educación superior como a la de "privatizar" las Instituciones de Educación Superior (IES). Posiblemente el peso superlativo dado en el discurso gubernamental a la idea de que la educación se resuelve mejor con calidad y de que hay que evaluar el desempeño de las IES midiendo la calidad de la educación que ofrecen, portó una carga conflictiva en la década de los 80 y sobre todo en la década de los 90. En la actualidad pareciera que se está asistiendo más a una especie de "consenso inestable" sobre la necesidad de formar también para la participación laboral y que hay que hacerlo con calidad pero sin perder 
de vista la importancia de principios propiamente educativos tales como el fomento de la curiosidad científica y humanística, en combinación con principios de formación integral.

En todos los países se espera que las instituciones de educación superior desempeñen un papel crucial en tiempos de la globalización y la sociedad del conocimiento, por lo que han estado en el centro de las reformas para provocar en ellas profundas transformaciones en sus prácticas y gobernanza. Se considera que están pobremente adaptadas a las nuevas condiciones de la globalización y la economía del conocimiento. Por ello, las políticas gubernamentales están impulsando cambios en áreas como el financiamiento, el aseguramiento de la calidad, la organización y el liderazgo, la administración de recursos humanos y las características del alumnado. Sin embargo, la experiencia indica que "entre más ha sido desarrollada esta tendencia, menos probable es que la implementación de políticas de reforma sea sencilla" (Musselin y Texeira, 2014: 2).

Asimismo debe tomarse en cuenta que el sistema de educación superior mexicano se convirtió en instrumento de contención social, ante la imposibilidad de que masas crecientes de jóvenes pudieran encontrar empleo digno en un contexto de crisis económica y crecimiento demográfico importante. "La demanda de trabajo y la imposibilidad de atenderla hizo que ésta se convirtiera en demanda educativa" contribuyendo así a ralentizar el efecto social de la crisis al ocurrir, en los hechos, una especie de "transferencia de responsabilidades" desde el sistema laboral hacia el sistema educativo (Martuscelli y Martínez, 2002: 4).

Otra cuestión a tomar en cuenta para el caso mexicano es que dicho papel de amortiguador social asignado al aparato educativo superior ocurrió a la par de una política de diversificación del sistema durante los años 90, que amplificó la enseñanza tecnológica a la vez que tendió a limitar la expansión del sector público universitario en favor del privado mediante un trato de laissez-faire por parte del Estado (Kent, 2005). Este trato permisivo se tradujo en la configuración de un sistema privado de educación superior extremadamente diferenciado en su calidad (Silas, 2005). Por un lado incluye instituciones de consolidado prestigio como el Instituto Tecnológico y de Estudios Superiores de Monterrey, Universidad Iberoamericana, Universidad de las Américas, Universidad Anáhuac y Universidad La Salle que ofrecen status y capital social además de formación académica, y por otro un amplio conjunto de instituciones "patito" (Rodríguez, 2006), de bajo costo, que ofrecen licenciaturas "de papel" (Igartúa, 2013) para satisfacer una parte de la creciente demanda de aquellos que se han refugiado en ellas luego de haber buscado, sin encontrar, lugar en las instituciones públicas.

\section{Metodología}

La información que alimentó esta investigación provino de la encuesta 'La reforma universitaria desde la perspectiva de los alumnos, UAMI 2014' (RUPA 2014), que fuera aplicada a 283 estudiantes seleccionados de todas las carreras y que conformaron una muestra probabilística estratificada con asignación de Neyman (Anderson et al., 1999: 890-892) (ver la distribución de la muestra tabla 1-1 del anexo). Los estratos fueron definidos cuidando que la información finalmente relevada representara a una población objetivo de 2596 estudiantes y que permitiera realizar estimaciones por sexo y por división académica: Ciencias Básicas e Ingeniería (CBI), Ciencias Sociales y Humanidades (CSH) y Ciencias Biológicas y de la Salud (CBS). La población enfocada incluyó a los alumnos de licenciatura regulares: activos (inscritos), con al menos $50 \%$ de créditos cubiertos, que han estado inscritos entre 6 y 18 trimestres, y que tienen un promedio numérico mayor a 7 en una escala de 0 a 10. 
Fueron construidos tres índices sumativos simples (Aprender a hacer, Aprender a aprender y Aprender a vivir juntos) (tabla 1), a fin de desentrañar la perspectiva de los alumnos de la UAMI respecto a su formación como preparación para su desenvolvimiento a lo largo de la vida y para, finalmente, participar en los mercados laborales. Cabe aclarar que, si bien en la mayoría de los casos el instrumento captó muchas más variables que las incluidas en los índices mencionados, las anotadas son las que saturaron satisfactoriamente. El análisis fue realizado de manera iterativa, descartando variables con escasa saturación, hasta que la medida de la adecuación muestral de Kaiser-Meyer-Olkin (Coeficiente KMO) del análisis factorial por componentes principales fuera mayor a 0.5, y la medida de confiabilidad, el coeficiente Alfa de Cronbach, registrara valores cercanos a 0.8 o superiores.

\section{Tabla 1. Variables incluidas en la construcción de índices basados en la encuesta RUPA}

\section{4}

\begin{tabular}{|c|c|c|}
\hline Índice & Variables seleccionadas & Escala \\
\hline $\begin{array}{l}\text { AH. Aprender } \\
\text { a hacer } \\
1124+1126+ \\
1127+1128+ \\
\text { IV } 1+\text { IV3 }\end{array}$ & $\begin{array}{l}\text { II } 24 \text { Mi formación en la universidad me brinda una sólida } \\
\text { base científica. } \\
\text { II } 26 \text { Mi formación en la universidad me brinda una sólida } \\
\text { base técnica. } \\
\text { II } 27 \text { Mi formación en la universidad me acerca al ejercicio } \\
\text { profesional. } \\
\text { II } 28 \text { Estoy aprendiendo cosas útiles para mi vida laboral. } \\
\text { IV1 El trabajo de campo o de laboratorio que he realizado } \\
\text { durante mi carrera me va a servir para tener un mejor } \\
\text { empleo. } \\
\text { IV3 Las prácticas profesionales de mi carrera sirven para } \\
\text { encontrar mejores empleos. }\end{array}$ & $6-24$ \\
\hline $\begin{array}{l}\text { AA. Aprender a } \\
\text { aprender } \\
\text { II } 33+1117+ \\
11111+11113 \\
+11114+ \\
11116+\mid 11121 \\
+11123+ \\
11124+11125\end{array}$ & $\begin{array}{l}\text { II } 33 \text { Estoy desarrollando habilidades para aprender por mi } \\
\text { propia cuenta. } \\
\text { III7 Busco información por mi cuenta aunque no me lo pidan } \\
\text { Ios profesores. } \\
\text { IIII1 Leo más de lo que requieren los programas de los } \\
\text { cursos. } \\
\text { III13 Comparto información académica en I nternet. } \\
\text { III14 Intercambio información con alumnos de otras } \\
\text { carreras. } \\
\text { III16 Asisto por iniciativa propia a eventos académicos } \\
\text { dentro de la universidad. } \\
\text { III23 Leo información académica en idioma extranjero. } \\
\text { III24 Aunque los profesores no lo requieran, realizo } \\
\text { resúmenes o esquemas conceptuales de mis lecturas. } \\
\text { III25 Leo constantemente sobre diversos temas. }\end{array}$ & $9-36$ \\
\hline $\begin{array}{l}\text { AV. Aprender a } \\
\text { vivir juntos } \\
119+120+ \\
121+122\end{array}$ & $\begin{array}{l}\text { I19 En mi carrera se fomenta la ética profesional. } \\
\text { I20 En mi carrera se fomenta la conciencia de } \\
\text { responsabilidad social. } \\
\text { I21 En mi carrera se discuten problemas nacionales. } \\
\text { I22 En mi carrera se fomentan los valores sociales. }\end{array}$ & 4- 16 \\
\hline
\end{tabular}

Fuente: Cálculos propios en STATA, con base en la encuesta 'La reforma universitaria desde la perspectiva de los alumnos', UAMI 2014. 


\section{III. ¿Qué tanto el estilo de enseñanza en la UAMI se apega a la idea de que la formación universitaria debería preparar a los estudiantes para su participación en el campo laboral y para la vida en general?}

Siguiendo las ideas de Delors et al. (1996) es posible pensar que la formación recibida en la UAMI es pertinente en la medida que sirve para que los estudiantes desarrollen habilidades en cuatro órdenes ligados a aprendizajes para la vida: aprender a conocer, aprender a hacer, aprender a vivir juntos y aprender a ser. El primer tipo de aprendizajes, "aprender a conocer", ligado al "aprender a aprender", implica el intercambio de información y la adquisición de conocimientos específicos y capacidades autodidactas que permiten seguir aprendiendo a lo largo de la vida. "Aprender a hacer" se refiere al desarrollo de competencias profesionales que permiten a los egresados insertarse exitosamente en los mercados de trabajo. "Aprender a vivir juntos" se refiere al respeto a la diversidad de pensamiento, premisa básica de la convivencia social y del trabajo en equipo. Finalmente, "aprender a ser" conlleva el fortalecimiento de la propia personalidad con autonomía de juicio y de responsabilidad personal. Estas habilidades, en esencia, constituyen el núcleo central de habilidades básicas necesarias para un buen desarrollo profesional.

La encuesta RUPA 2014 da idea de que la población estudiantil tiene un claro patrón de prioridades que, de alguna manera, la formación universitaria debería tomar en cuenta. Desde la perspectiva de los estudiantes, en el plano de mayor importancia se encuentran los aspectos relacionados con el aprender a hacer y a conocer $y$, en segundo plano, los referidos a cuestiones humanistas del aprender a vivir juntos y del aprender a ser.

La tabla 2 muestra que si bien todos los aspectos indagados de la formación arrojaron puntajes medios por encima de la mitad de la escala (entre 3.6 y 4.5 puntos en una escala que puede adoptar valores desde 1 hasta 7), el análisis de los límites de confianza (tabla 2-1 del anexo) muestra que en los primeros lugares de importancia están el recibir una formación para el trabajo (4.5 puntos promedio) y para la solución de problemas sociales (4.5 puntos); después siguen la formación para resolver preguntas científicas y tecnológicas (4.1 puntos), y la formación en valores y ética profesionales (4.1 puntos); y, al final, menos importantes resultaron la formación con sentido de responsabilidad social (3.7 puntos) y los aspectos relacionados con la formación para ser mejores personas y ciudadanos (3.6 puntos) y para fomentar una sociedad más justa (3.6 puntos). 


\section{Tabla 2. Puntajes promedio en la pregunta ¿Qué considera más importante en su formación universitaria?}

\begin{tabular}{|l|c|c|c|c|c|c|}
\hline \multicolumn{1}{|c|}{$\begin{array}{c}\text { ¿Qué considera más importante en su formación } \\
\text { universitaria? Formar: }\end{array}$} & UAMI & Masc. & Fem. & CBI & CSH & CBS \\
\hline para ofrecer soluciones a problemas sociales & 4.5 & 4.5 & 4.5 & 4.5 & $4.4^{\ddagger}$ & 4.7 \\
\hline para el trabajo & 4.5 & 4.4 & 4.6 & 4.5 & 4.6 & 4.4 \\
\hline para resolver preguntas científicas y tecnológicas & 4.1 & 4.2 & 4.1 & $4.8^{\#}$ & $3.7^{\ddagger}$ & 4.6 \\
\hline en valores y ética profesionales & 4.1 & 4.0 & $4.2^{\star}$ & 4.0 & 4.1 & 4.0 \\
\hline con sentido de responsabilidad social & 3.7 & 3.6 & 3.7 & 3.8 & $4.0^{\ddagger}$ & $3.1^{\ddagger}$ \\
\hline para ser mejores personas y ciudadanos & 3.6 & 3.6 & 3.6 & 3.3 & 3.6 & $3.7^{\ddagger}$ \\
\hline para fomentar una sociedad más justa & 3.6 & 3.7 & 3.5 & $2.8^{\#}$ & $3.9^{\ddagger}$ & $3.4^{\ddagger}$ \\
\hline
\end{tabular}

* Diferencias estadísticamente significativas entre hombres y mujeres, con 95\% de confianza. La tabla 2-1 del anexo muestra los intervalos de confianza.

\# Diferencias estadísticamente significativas entre CBI y CSH, con 90\% de confianza.

${ }^{*}$ Diferencias estadísticamente significativas entre CSH y CBS, con $90 \%$ de confianza.

₹ Diferencias estadísticamente significativas entre CBS y CBI, con $90 \%$ de confianza.

Fuente: Cálculos propios en STATA, con base en la encuesta 'La reforma universitaria desde la perspectiva de los alumnos', UAMI 2014.

Abreviaturas: CBI, Ciencias Básicas e Ingeniería; CSH, Ciencias Sociales y Humanidades; CBS, Ciencias Biológicas y de la Salud.

Patrones similares se dan tanto entre hombres como entre mujeres con algún matiz. Las mujeres tienden a conceder ligeramente más importancia a la formación en valores y ética profesionales que los hombres. A nivel de las divisiones académicas también se verifica un patrón similar al general del conjunto UAMI, pero con algunas especificidades.

La formación para la solución de preguntas científicas y tecnológicas es un aspecto de la mayor importancia para CBI y CBS, mientras que para CSH está entre los aspectos de menor importancia. Probablemente las ideas de los alumnos de CSH han sido influidas por aquella de que "la ciencia" sólo concierne las llamadas "ciencias de la naturaleza" o "ciencias duras", por lo que en conjunto tienden a no conceder calidad de ciencia a lo social. Otras especificidades tienen que ver con que CBS y CBI se distinguen porque la primera concede la menor importancia a la formación con sentido de responsabilidad social, y la segunda hace lo mismo en cuanto a formar para una sociedad más justa.

Finalmente, es claro que si bien el tema de la formación para el trabajo se encuentra entre los tres de mayor importancia para las tres divisiones, en CSH ocupa el primer lugar mientras que en CBI y CBS es relegado a un segundo y tercer lugar respectivamente, después de la formación para resolver preguntas científicas y tecnológicas y para resolver problemas sociales. Sorprende que este último aspecto haya resultado más importante para los estudiantes de CBS que para los de CSH y CBI. Es probable que la aproximación de una parte de los alumnos de CBS a las comunidades agropecuarias, forestales y acuícolas donde realizan sus prácticas de campo ayude a entender esa mayor importancia.

La misma encuesta también aporta elementos para realizar una aproximación a saber qué tanto la percepción de los alumnos indica que la formación recibida se aproxima a lo planteado por Delors et al. (1996) en tres de sus pilares: aprender a hacer, aprender a conocer y aprender a vivir juntos. La tabla 3 ofrece tres índices que permiten un acercamiento a saber qué tanto están percibiendo los alumnos que su formación les está propiciando aprendizajes en estos tres aspectos. Los puntajes promedio de dos de estos 
índices (AH y AV) se ubicaron en el extremo superior de sus correspondientes escalas; en cambio el índice AA que aporta elementos del aprender a conocer registró un puntaje promedio ubicado cerca de la mitad de la escala.

Tabla 3. Valores medios en índices de formación para la vida, 2014

\begin{tabular}{|l|r|r|r|r|r|r|r|}
\hline \multirow{2}{*}{ Índice } & \multirow{2}{*}{ Escala } & \multirow{2}{*}{ UAMI } & \multicolumn{2}{|c|}{ Sexo } & \multicolumn{4}{c|}{ División } \\
& & & Masc. & \multicolumn{1}{c|}{ Fem. } & CBI & CSH & CBS \\
\hline Aprender a hacer (AH) & $6-24$ & 20.2 & 20.0 & 20.4 & 21.1 & $19.2^{*}$ & $20.9^{\#}$ \\
\hline Aprender a aprender (AA) & $9-36$ & 24.3 & 24.4 & 24.3 & 23.9 & 24.5 & 24.3 \\
\hline Aprender a vivir juntos (AV) & $4-16$ & 13.0 & 13.0 & 13.0 & 12.8 & $13.6^{*}$ & $12.4^{\#}$ \\
\hline
\end{tabular}

\# Diferencias estadísticamente significativas respecto a CBI, con 90\% de confianza.

\# Diferencias estadísticamente significativas respecto a $\mathrm{CSH}$, con $90 \%$ de confianza.

Fuente: Cálculos propios en STATA, con base en la encuesta 'La reforma universitaria desde la perspectiva de los alumnos', UAMI 2014.

\section{Aprender a hacer}

Ya se vio que para los estudiantes de la UAMI, independientemente de la división académica en la que se inscriben, la formación para el trabajo es de la mayor importancia. En este sentido, la información extraída de la encuesta RUPA 2014 muestra que los estudiantes de la UAMI tienen una alta valoración de la formación que reciben porque piensan que les está ofreciendo elementos que les pueden servir para insertarse exitosamente en el campo laboral, como los relacionados con la construcción de una sólida base científica y técnica. Los resultados del índice Aprender a hacer arrojaron un puntaje promedio elevado, 20.2 en una escala que va de 6 a 24 puntos (tabla 3), y tanto hombres como mujeres coincidieron en esta alta valoración. Sin embargo, a nivel de las divisiones, los alumnos de CBI fueron los que mayor puntaje registraron (21.1), seguidos por los de CBS (20.9). Los de CSH fueron los que registraron el puntaje estadísticamente más bajo aunque no deja de ser elevado (19.2 puntos). Estas diferencias divisionales pueden ser resultado de que en varias carreras de CBI y CBS la orientación profesional busca la aplicación de conocimientos y la experimentación en ambientes prácticos.

La tabla 4 muestra cómo se distribuyeron las respuestas en cada una de las variables involucradas en el índice. En general, la mayor parte de los alumnos -alrededor del 80\% o más- estuvo de acuerdo en que la universidad les está brindando una sólida base científica y tecnológica, en que su formación los acerca al ejercicio profesional, y en que están aprendiendo cosas útiles para la vida laboral. No obstante, sobresalen los elevados porcentajes de respuestas desfavorables relacionadas con el trabajo de campo o de laboratorio y las prácticas profesionales. Casi un $40 \%$ no estuvo de acuerdo en que el trabajo de campo o de laboratorio realizado durante su carrera le vaya a servir para obtener un mejor empleo, o no ve que existan estas actividades en su carrera o no las ha realizado, y más de un $50 \%$ no estuvo de acuerdo en que las prácticas profesionales sirvan para encontrar mejores empleos o no ve que haya prácticas profesionales o no las ha realizado. Un análisis a nivel de las divisiones reveló que el porcentaje de percepciones desfavorables sobre el trabajo de campo o laboratorio y sobre las prácticas profesionales fue mayor en $\mathrm{CSH}$ (56.1\% y $61.2 \%$, respectivamente), seguido por CBI (25.9\% y $52.3 \%$ ) y finalmente CBS (12\% y $37.2 \%)$. 
Estos datos sorprenden porque se trata de jóvenes con poco más de 24 años promedio, que tienen casi $68 \%$ de créditos cubiertos y que han acumulado casi 13 trimestres de actividad real (trimestres en que se han inscrito a UEA) en la UAMI. Revelan dudas sobre qué tanto, realmente, la formación recibida estará recurriendo a estrategias útiles para que los alumnos puedan incursionar exitosamente en el campo laboral. Los trabajos de campo y de laboratorio y las prácticas profesionales son espacios concretos donde las experiencias de los estudiantes se aproximan a la realidad de los mercados profesionales e incluso significan la primera incursión en los mismos, aunque a veces sólo como espectadores, de modo que en muchos casos podrían constituir verdaderos apoyos para superar algunas de las barreras a la entrada de dichos mercados.

Tabla 4. Distribución porcentual en los componentes del índice AH, Aprender a hacer. Encuesta RUPA 2014.

\begin{tabular}{|l|c|c|c|c|}
\hline \multicolumn{1}{|c|}{ Aprender a hacer } & Desacuerdo & Acuerdo & $\begin{array}{c}\text { No hay, } \\
\text { no he } \\
\text { realizado }\end{array}$ & Total \\
\hline $\begin{array}{l}\text { Mi formación en la universidad me brinda } \\
\text { una sólida base científica. }\end{array}$ & 15.4 & 84.6 & na & 100.0 \\
\hline $\begin{array}{l}\text { Mi formación en } \\
\text { la universidad } \\
\text { me brinda una } \\
\text { sólida base } \\
\text { técnica }\end{array}$ & 22.5 & 77.5 & na \\
\hline $\begin{array}{l}\text { Mi formación en la universidad me acerca } \\
\text { al ejercicio profesional. }\end{array}$ & 11.4 & 88.6 & na & 100.0 \\
\hline $\begin{array}{l}\text { Estoy aprendiendo cosas útiles para mi } \\
\text { vida laboral. }\end{array}$ & 14.1 & 84.2 & na & 100.0 \\
\hline $\begin{array}{l}\text { El trabajo de campo o de laboratorio que } \\
\text { he realizado durante mi carrera me va a } \\
\text { servir para tener un mejor empleo. }\end{array}$ & 8.6 & 62.2 & 29.2 & 100.0 \\
\hline $\begin{array}{l}\text { Las prácticas profesionales de mi carrera } \\
\text { sirven para encontrar mejores empleos. }\end{array}$ & 9.8 & 47.5 & 42.7 & 100.0 \\
\hline
\end{tabular}

Fuente: Cálculos propios en STATA, con base en la encuesta 'La reforma universitaria desde la perspectiva de los alumnos'. UAMI 2014.

La elevada valoración que tienen los estudiantes de la formación que reciben coincide con sus igualmente elevadas expectativas de desempeñarse en ocupaciones de alta jerarquía y como profesionistas.

La tabla 5 muestra que casi la cuarta parte (23.3\%) piensa que su formación en la UAMI debería servirle para desempeñarse como empresario, director o subdirector; $38.7 \%$, como consultor, asesor o profesionista con ejercicio libre de la profesión, y $28.2 \%$, como jefe o supervisor. Sólo menos del 10\% del alumnado manifestó expectativas pesimistas al considerar que sus estudios deberían servirle para ser empleado sin personal a su cargo (7.9\%) u obrero (0.5\%), o que no le ayudarán a obtener empleo (1.3\%). 
Tabla 5. Pienso que mis estudios deberían servirme para tener al menos una jerarquía de:

\begin{tabular}{|l|c|}
\hline \multicolumn{1}{|c|}{ J erarquías } & $\%$ \\
\hline Consultor, asesor o profesionista (ejercicio libre de la profesión) & 38.7 \\
\hline J efe o supervisor & 28.2 \\
\hline Director, subdirector & 14.5 \\
\hline Empresario & 8.8 \\
\hline Empleado sin personal a mi cargo & 7.9 \\
\hline Creo que mis estudios no me ayudarán a tener trabajo & 1.3 \\
\hline Obrero & 0.5 \\
\hline Total & 100 \\
\hline
\end{tabular}

Fuente: Cálculos propios en STATA, con base en la encuesta 'La reforma universitaria desde la perspectiva de los alumnos', UAMI 2014.

\section{Aprender a aprender}

El valor medio del índice Aprender a aprender mostrado en la tabla 3 no es tan elevado como el de los otros dos índices de formación para la vida; de hecho se encuentra casi a la mitad de la escala, 24.3 puntos en una gradación que va de 9 a 36 puntos. Esto da idea de que buena parte de los estudiantes no perciben que la universidad esté formándolos para seguir aprendiendo a lo largo de la vida. No hay diferencias estadísticamente significativas entre hombres y mujeres; ambos sexos registraron puntajes promedio similares al mencionado para el conjunto de la UAMI (24.4 y 24.3 puntos, respectivamente). A nivel de las divisiones, la evidencia estadística igualmente muestra que no existen diferencias: las tres registraron puntajes del orden de los 24 puntos. No obstante, a juzgar por el dato puntual registrado en CBI (23.9 puntos) cabe la posibilidad de que una indagación basada en un tamaño muestral más robusto arroje que los estudiantes de CBI tienen opiniones menos favorables en las variables que constituyeron este índice de aprender a aprender.

La tabla 6 ofrece información detallada sobre los componentes del índice aprender a aprender. Si bien sólo cerca del 10\% de los alumnos de la UAMI dijo no estar de acuerdo con la afirmación que alude explícitamente a la formación de habilidades para aprender de manera autodidacta, el resto de los reactivos registró opiniones desfavorables con mucha más frecuencia. Del total de alumnos en la UAMI objeto de este estudio, un tercio (34.4\%) no suele buscar información más allá de la solicitada por sus profesores; más de un tercio (38.6\%) manifiesta no tener constancia en la lectura y tampoco suele asistir a eventos académicos dentro de la misma universidad (42.5\%); la mitad prácticamente no lee más de lo que requieren los cursos (50.4\%), tampoco acostumbra compartir información académica en Internet (51.7\%), ni leer información académica en idioma extranjero (55.2 \%); porcentajes aún mayores no suelen hacer resúmenes o esquemas de sus lecturas (58.4\%), o intercambiar información con pares de otras carreras (59.7\%). Estos datos insinúan la existencia de cierta dificultad en el proceso de formación en la UAMI. Posiblemente se trate de manifestaciones fragmentarias de un problema de doble vía. Por un lado podría estar asociado a una cultura estudiantil de orientación conformista hacia el aprendizaje pero, por otro, también habría que preguntarse qué tanto la práctica docente está estimulando el desarrollo de habilidades para que el alumnado siga aprendiendo a lo largo de la vida. 
Tabla 6. Distribución porcentual en los componentes del índice AA, Aprender a aprender. Encuesta RUPA 2014.

\begin{tabular}{|c|c|c|c|}
\hline Aprender a aprender & $\begin{array}{l}\text { Nunca o } \\
\text { a veces }\end{array}$ & $\begin{array}{l}\text { Muchas } \\
\text { veces o } \\
\text { siempre }\end{array}$ & Total \\
\hline $\begin{array}{l}\text { Estoy desarrollando habilidades para aprender por mi propia } \\
\text { cuenta* }\end{array}$ & 8.8 & 91.2 & 100 \\
\hline $\begin{array}{l}\text { Busco información por mi cuenta aunque no me lo pidan los } \\
\text { profesores. }\end{array}$ & 34.4 & 65.6 & 100 \\
\hline Leo más de lo que requieren los programas de los cursos. & 50.4 & 49.6 & 100 \\
\hline Comparto información académica en Internet. & 51.7 & 48.3 & 100 \\
\hline Intercambio información con alumnos de otras carreras. & 59.7 & 40.3 & 100 \\
\hline $\begin{array}{l}\text { Asisto por iniciativa propia a eventos académicos dentro de la } \\
\text { universidad. }\end{array}$ & 42.5 & 57.5 & 100 \\
\hline Leo información académica en idioma extranjero. & 55.2 & 44.8 & 100 \\
\hline $\begin{array}{l}\text { Aunque los profesores no lo requieran, realizo resúmenes o } \\
\text { esquemas conceptuales de mis lecturas. }\end{array}$ & 58.4 & 41.6 & 100 \\
\hline Leo constantemente sobre diversos temas. & 38.6 & 61.4 & 100 \\
\hline
\end{tabular}

* La redacción del reactivo refiere a la siguiente variante en las categorías: desacuerdo (8.9\%) y acuerdo (91.1\%).

Fuente: Cálculos propios en STATA, con base en la encuesta 'La reforma universitaria desde la perspectiva de los alumnos', UAMI 2014.

\section{Aprender a vivir juntos}

El fomento de los valores éticos y sociales, y de conciencia y responsabilidad sociales son componentes fundamentales de una formación para propiciar mayor convivencia colectiva. El índice Aprender a vivir juntos ofrece elementos para dimensionar qué tanto los alumnos de la UAMI perciben que su formación, en los hechos, está siendo orientada por esta idea. La tabla 3 mostró que sus apreciaciones son altamente positivas. El valor medio del índice registró 13 puntos en una escala que va de 4 a 16. No hubo diferencias estadísticamente significativas entre hombres y mujeres; ambos sexos registraron 13 puntos promedio. Al nivel de las divisiones se distinguieron los alumnos de CSH porque fueron los que mejor puntuación concedieron a su formación en este sentido (13.6 puntos), en comparación con CBI (12.8 puntos) y CBS (12.4 puntos).

Todos los reactivos componentes del índice AV sin excepción registraron elevada frecuencia de opiniones favorables (tabla 7). Más del $80 \%$ de los alumnos estuvo de acuerdo en que en su carrera se fomenta la ética profesional, la conciencia de responsabilidad social y los valores sociales. Asimismo, casi tres cuartos (74.1\%) consideró que en su carrera se discuten problemas nacionales. Entonces, cabe decir que la formación profesional se encuentra, dadas estas percepciones, permeada por un fuerte concepto de sociabilidad y de conciencia social, de modo que incluso podría constituir uno de los activos formativos de la institución. 
Tabla 7. Distribución porcentual en los componentes del índice AV, Aprender a vivir juntos. Encuesta RUPA 2014.

\begin{tabular}{|l|c|c|c|}
\hline \multicolumn{1}{|c|}{ Aprender a vivir juntos } & Desacuerdo & Acuerdo & Total \\
\hline En mi carrera se fomenta la ética profesional. & 15.9 & 84.1 & 100 \\
\hline $\begin{array}{l}\text { En mi carrera se fomenta la conciencia de } \\
\text { responsabilidad social. }\end{array}$ & 15.4 & 84.6 & 100 \\
\hline En mi carrera se discuten problemas nacionales. & 25.9 & 74.1 & 100 \\
\hline En mi carrera se fomentan los valores sociales. & 19.8 & 80.2 & 100 \\
\hline
\end{tabular}

Fuente: Cálculos propios en STATA, con base en la encuesta 'La reforma universitaria desde la perspectiva de los alumnos', UAMI 2014.

\section{Conclusiones}

La UAM -y en particular la unidad Iztapalapa- ha realizado ajustes formales a su modelo educativo durante los últimos diez años a fin de reforzar la pertinencia del mismo. Dichos ajustes han quedado plasmados en las denominadas Políticas Generales, Políticas Operacionales, y Políticas Operativas de Docencia. En las mismas se asientan cambios asociados a nuevas lógicas de formación universitaria bajo la idea de producir una mejor vinculación de la misma con los requerimientos de los mercados laborales, de fortalecer el autodidactismo del alumnado, y de elevar las posibilidades de convivencia por vía de la educación integral, humanista y orientada a la solución de problemas sociales y ambientales. Esta investigación aporta elementos para dirimir qué tanto estos lineamientos formales están permeando la práctica educativa de la UAMI. Exploró la relación de coherencia que, desde la perspectiva de los estudiantes, se da entre sus expectativas sobre el para qué de la educación universitaria y la utilidad concreta de la formación que reciben en la UAMI. Encontró que la población estudiantil tiene un claro patrón de prioridades sobre las cuales la institución necesita seguir reflexionando para tomar decisiones a la hora de diseñar su oferta educativa. El análisis de información proveniente de la encuesta RUPA 2014 muestra que, para los estudiantes, en el plano de la mayor importancia se encuentra el que la formación recibida sea útil para "aprender a hacer" y "aprender a conocer", es decir, que fomente la adquisición de conocimientos y competencias útiles para la participación laboral, la solución de problemas y para seguir aprendiendo a lo largo de la vida. En contraste, las cuestiones formativas humanistas del "aprender a vivir juntos", como el fomento de una sociedad más justa y la formación con sentido de responsabilidad social son aspectos que el alumnado relega a un segundo plano de prioridades. Patrones similares se dan tanto entre hombres como entre mujeres, pero con matices, ya que las mujeres tienden a conceder ligeramente más importancia a la formación en valores y ética profesionales que los hombres. A nivel de las divisiones académicas también se verifica un patrón similar al del conjunto UAMI, pero con algunas especificidades. La formación para la solución de preguntas científicas y tecnológicas es un aspecto de la mayor importancia para el alumnado de CBI y CBS, mientras que para CSH está entre los aspectos de menor importancia. Probablemente los alumnos de CSH han sido influidos por aquella idea de que "la ciencia" sólo es un asunto de las Ilamadas "ciencias de la naturaleza" o "ciencias duras", por lo que en conjunto tienden a no conceder calidad de ciencia a lo social.

El análisis de tres índices sintéticos sobre las percepciones de los estudiantes en cuanto a la formación concreta que reciben en la UAMI da idea de que la labor educativa de la universidad se corresponde, en gran medida, con las expectativas estudiantiles de "aprender a hacer" y "aprender a convivir", más no tanto con las de "aprender a aprender". 
Gran parte de los estudiantes piensan que sus estudios les ayudarán a tener una buena inserción laboral, a la vez que tienen una alta valoración de la formación que reciben. Consideran que ésta les está dotando de elementos útiles para el trabajo, tales como los relacionados con la construcción de sólidas bases científicas y técnicas. No obstante, el alto porcentaje de percepciones desfavorables respecto al trabajo de campo o laboratorio y a las prácticas profesionales revela dudas sobre qué tanto, realmente, la formación recibida estará recurriendo a estrategias útiles para que los alumnos puedan incursionar exitosamente en el campo laboral. Los trabajos de campo y de laboratorio y las prácticas profesionales son espacios concretos en los que las experiencias de los estudiantes se aproximan a la realidad de los mercados profesionales. Incluso podrían ser la primera incursión en los mismos, de modo que en muchos casos podrían constituir verdaderos apoyos para superar algunas de las barreras a la entrada a dichos mercados.

Las valoraciones de los estudiantes también son altamente positivas en cuanto a los aportes de su formación para aprender a convivir, relacionados con aspectos como el fomento de la responsabilidad y los valores sociales, y la ética profesional. No obstante, sus apreciaciones dan idea de que la universidad no lo está haciendo tan bien en cuanto a la formación para seguir aprendiendo a lo largo de la vida, particularmente en aspectos ligados al autodidactismo tales como la lectura en idioma extranjero, el intercambio interdisciplinar y la automotivación para realizar actividades de aprendizaje. Estas son cuestiones importantes en las cuales convendría que la universidad pusiera atención en el futuro.

Estos resultados contradicen una de las hipótesis con que arrancó la investigación, la cual planteaba la posibilidad de que los alumnos valoraran desfavorablemente la pertinencia de la formación recibida en cuanto a los elementos ofrecidos por ésta para una buena inserción al mercado de trabajo. 


\section{Anexo}

Tabla 1.1. Muestras obtenidas por división, sexo y plan de estudios. UAMI

\begin{tabular}{|c|c|c|}
\hline División & Plan de estudios & Total \\
\hline \multirow{10}{*}{$\mathrm{CBI}$} & 22 Licenciatura en Ingeniería Biomédica & 7 \\
\hline & 23 Licenciatura en Ingeniería Hidrológica & 3 \\
\hline & 24 Licenciatura en Ingeniería Química & 4 \\
\hline & 25 Licenciatura en Ingeniería en Energía & 5 \\
\hline & 26 Licenciatura en Física & 6 \\
\hline & 27 Licenciatura en Ingeniería Electrónica & 6 \\
\hline & 28 Licenciatura en Matemáticas & 5 \\
\hline & 29 Licenciatura en Química & 3 \\
\hline & 30 Licenciatura en Computación & 7 \\
\hline & Total & 46 \\
\hline \multirow{12}{*}{$\mathrm{CSH}$} & 37 Licenciatura en Administración & 39 \\
\hline & 38 Licenciatura en Antropología Social & 8 \\
\hline & 39 Licenciatura en Letras Hispánicas & 10 \\
\hline & 40 Licenciatura en Filosofía & 9 \\
\hline & 41 Licenciatura en Economía & 11 \\
\hline & 42 Licenciatura en Sociología & 15 \\
\hline & 43 Licenciatura en Historia & 11 \\
\hline & 44 Licenciatura en Lingüística & 5 \\
\hline & 45 Licenciatura en Ciencia Política & 16 \\
\hline & 46 Licenciatura en Psicología Social & 21 \\
\hline & 121 Licenciatura en Geografía Humana & 6 \\
\hline & Total & 151 \\
\hline \multirow{7}{*}{ CBS } & 52 Licenciatura en Biología & 24 \\
\hline & 53 Licenciatura en Biología Experimental & 11 \\
\hline & 54 Licenciatura en Hidrobiología & 7 \\
\hline & 55 Licenciatura en Producción Animal & 4 \\
\hline & 56 Licenciatura en Ingeniería Bioquímica Industrial & 23 \\
\hline & 57 Licenciatura en Ingeniería de los Alimentos & 17 \\
\hline & Total & 86 \\
\hline & Total UAMI & 283 \\
\hline
\end{tabular}


Tabla 2.1 Límites de confianza de los valores medios en la pregunta ¿Qué considera más importante en su formación universitaria?

\begin{tabular}{|c|c|c|c|c|c|c|c|c|c|c|c|c|}
\hline \multirow{3}{*}{$\begin{array}{c}\text { ¿Qué considera } \\
\text { más importante } \\
\text { en su formación } \\
\text { universitaria? } \\
\text { Formar: }\end{array}$} & \multirow{3}{*}{$\begin{array}{c}\text { UAMI } \\
\text { LI }\end{array}$} & & \multicolumn{4}{|c|}{ Sexo } & \multicolumn{6}{|c|}{ División } \\
\hline & & \multicolumn{2}{|c|}{ Masc. } & \multicolumn{2}{|c|}{ Fem. } & \multicolumn{2}{|c|}{$\mathrm{CBI}$} & \multicolumn{2}{|c|}{$\mathrm{CSH}$} & \multicolumn{2}{|c|}{ CBS } & \multirow[b]{2}{*}{ LS } \\
\hline & & LS & $\mathrm{LI}$ & LS & LI & LS & $\mathrm{LI}$ & LS & $\mathrm{LI}$ & LS & $\mathrm{LI}$ & \\
\hline $\begin{array}{l}\text { V2c para ofrecer } \\
\text { soluciones a } \\
\text { problemas sociales }\end{array}$ & 4.43 & 4.55 & 4.43 & 4.62 & 4.39 & 4.54 & 4.37 & 4.66 & 4.27 & 4.44 & 4.62 & 4.82 \\
\hline V2a para el trabajo & 4.46 & 4.61 & 4.32 & 4.57 & 4.51 & 4.71 & 4.32 & 4.73 & 4.48 & 4.70 & 4.31 & 4.58 \\
\hline $\begin{array}{l}\text { V2e para resolver } \\
\text { preguntas } \\
\text { científicas y } \\
\text { tecnológicas }\end{array}$ & 4.05 & 4.20 & 4.11 & 4.32 & 3.96 & 4.16 & 4.62 & 5.00 & 3.57 & 3.77 & 4.51 & 4.75 \\
\hline $\begin{array}{l}\text { V2b en valores y } \\
\text { ética profesional }\end{array}$ & 4.04 & 4.16 & 3.86 & 4.06 & 4.12 & 4.27 & 3.87 & 4.22 & 4.06 & 4.23 & 3.94 & 4.13 \\
\hline $\begin{array}{l}\text { V2g con un sentido } \\
\text { de responsabilidad } \\
\text { social }\end{array}$ & 3.63 & 3.76 & 3.53 & 3.72 & 3.66 & 3.83 & 3.69 & 3.99 & 3.89 & 4.05 & 3.02 & 3.24 \\
\hline $\begin{array}{l}\text { V2d para ser } \\
\text { mejores personas y } \\
\text { ciudadanos }\end{array}$ & 3.51 & 3.63 & 3.49 & 3.68 & 3.48 & 3.63 & 3.20 & 3.49 & 3.48 & 3.64 & 3.57 & 3.79 \\
\hline $\begin{array}{l}\text { V2f para fomentar } \\
\text { una sociedad más } \\
\text { justa }\end{array}$ & 3.53 & 3.65 & 3.58 & 3.75 & 3.46 & 3.62 & 2.70 & 2.99 & 3.80 & 3.95 & 3.31 & 3.54 \\
\hline
\end{tabular}

Abreviaturas: LI Límite inferior con 90\% de confianza; LS Límite superior con 90\% de confianza. Fuente: Cálculos propios en STATA, con base en la encuesta 'La reforma universitaria desde la perspectiva de los alumnos', UAMI 2014.

\section{Referencias bibliográficas}

Anderson, D.; Sweeney, D.; Williams, T. (1999). Estadística para Administración y Economía. México, D.F.: International Thomson Editores.

ANUIES (1973). Estudio sobre la demanda de educación de nivel medio superior y nivel superior (primer ingreso) en el país y proposiciones para su solución. México D.F.

ANUIES (1981). Plan Nacional de Educación Superior. Lineamientos generales para el periodo 1981-1991. Revista de Educación Superior, 10 (39). http://publicaciones.anuies.mx/ revista/39

Congreso de la Unión (1978, 29 de diciembre). Ley de Coordinación de la Educación Superior. Diario Oficial de la Federación. México D.F.: Cámara de Diputados.

De Garay, A. (2000). Los actores desconocidos. Una aproximación al conocimiento de los estudiantes. México D.F.: ANUIES.

De Garay, A. (2011). Mis estudios y propuestas sobre los jóvenes universitarios mexicanos. Trabajo presentado para obtener la Cátedra Rafael Cordera Campos de la UAMAzcapotzalco. http://www.fundacionucol.org/wp-content/uploads/2013/09/Estudios-ypropuestas-Adrian-de-Garay.pdf 
De la Orden, A. (2007). Evaluación de la calidad de la educación. Un modelo sistémico como base para la construcción de un sistema de indicadores. En INEE. Conceptos, Metodologías y Experiencias para la Construcción de Sistemas de Indicadores Educativos. Memoria del Primer Seminario Internacional de Indicadores Educativos. México D.F.: Instituto Nacional para la Evaluación de la Educación, pp. 5-22.

Delors, J. (coord.) (1996). La educación encierra un tesoro. París: Ediciones UNESCO

De Vries, W. (coord.) (2005). Calidad, Eficiencia y Evaluación de la Educación Superior. España: Netbiblo S. L.

Gago, A. (2002). Apuntes acerca de la evaluación educativa. México D.F.: SEP. Igartúa, S. (2013, marzo 7). Licenciaturas de papel. Proceso, No.1896.

Kent, R. (2005). La dialéctica de la esperanza y la desilusión en políticas de educación superior en México. Revista de la Educación Superior, 34-2(134), abril-junio, pp. 63-79. México D.F.: NUIES.

Latapí, P. (1980). Análisis de un sexenio de educación en México, 1970-1976. México D.F.: Nueva Imagen (Apud Rojas, 2005).

López, R.; Casillas, M.; González, O. (2000). Una historia de la UAM: sus primeros 25 años. México D.F.: UAM.

Martínez, F.; Robles, H.; Hernández, J.; Zendejas, L.; Pérez, M. (2007). Propuesta y experiencias para desarrollar un sistema nacional de indicadores educativos. México D.F.: Instituto Nacional para la Evaluación de la Educación, pp 280.

Martuscelli, J. \& Martínez, C. (2002). Problemas de la Pertinencia de la Educación Superior en el Mercado Laboral. Memoria del Tercer Congreso Nacional y Segundo Internacional Retos y Experiencias de la Universidad, 6-9 de noviembre, Toluca, México: Universidad Autónoma del Estado de México.

Musselin, C. \& Teixeira, P. (Eds.) (2014). Reforming Higher Education. Public Policy Design and Implementation. Springer Netherlands.

Presidencia de la República (2013). Plan nacional de desarrollo 2013-2018. México, D.F.: Autor.

Robles, H. (coord.); Hernández, J.; Peralta, Y., et al. (2012). Panorama educativo de México 2010. Indicadores del Sistema Educativo Nacional. Educación básica y media superior. México D.F.: Instituto Nacional para la Evaluación de la Educación (INEE).

Robles, H. (Coord.); Pérez, M.; Medrano, M., et al. (2014). Panorama educativo de México 2013. Indicadores del Sistema Educativo Nacional. Educación básica y media superior. México D.F.: Instituto Nacional para la Evaluación de la Educación (INEE).

Rodríguez, R. (2006). Relaciones peligrosas. Segunda parte. Campus Milenio. No.166. http:// www.ses.unam.mx/publicaciones/articulos.php?proceso=visualiza\&idart=274

Rodríguez, J.; Hernández, J. \& Mecalco, M. (2007). Algunos aspectos de la formación profesional y la situación laboral de los egresados de la UAMI, 1998-2002, México, D.F.: UAMI. 
Rubio, Julio (2006). La política educativa y la educación superior en México 1995-2006. Un balance. México, D.F.: FCE.

Santos, A. \& Delgado, A. (2011). Capítulo 1. Consideraciones sobre la obligatoriedad y la composición de la educación media superior. En INEE. La Educación Media Superior en México. México D.F.: Instituto Nacional para la Evaluación de la Educación, pp. 13-44.

Saucedo C.; Guzmán, C.; Sandoval E., Galaz J. (2013). Estudiantes, maestros y académicos en la investigación educativa. Tendencias, aportes y debates 2002-2011. México D.F.: ANUIES y COMIE

SEP (2001). Programa Nacional de Educación 2001-2006. México D.F.: Secretaría de Educación Pública.

SEP (2007). Programa Sectorial de Educación 2007-2012. México D.F.: Secretaría de Educación Pública.

SEP (2013, 13 de noviembre). Programa Sectorial de Educación 2013-2018. Diario Oficial de la Federación. México D.F.: Cámara de Diputados.

Silas, J. (2005). Realidades y tendencias de la educación superior privada mexicana. Perfiles Educativos. 27(109-110), julio-diciembre, pp. 7-37. México D.F.: Instituto de Investigaciones sobre la Universidad y la Educación.

Taccari, D. (2007). Marco conceptual y metodológico del sistema regional de indicadores educativos de la Unesco Santiago en el marco de EPT / Prealc. En INEE. Conceptos, Metodologías y Experiencias para la Construcción de Sistemas de Indicadores Educativos. Memoria del Primer Seminario Internacional de Indicadores Educativos. México D.F.: Instituto Nacional para la Evaluación de la Educación, 23-36.

Unesco (1998). Declaración Mundial sobre la Educación Superior en el Siglo XXI: Visión y Acción. En Universidad de Guadalajara (2004). Temas de educación superior. Guadalajara, México: Autor, pp. 7-18.

Unesco-Orealc (2007). Situación educativa de América Latina y el Caribe: garantizando la educación de calidad para todos. Santiago, Chile: Unesco, Oficina Regional de Educación para América Latina y el Caribe.

Velarde, D. \& Camarena, B. (2009). Educación superior y mercado laboral: vinculación y pertinencia social ¿Por qué y para qué? Memoria del X Congreso Nacional de Investigación Educativa. México: Consejo Mexicano de Investigación Educativa (Comie).

\section{Bases de datos}

Hernández J. y Rodríguez J. (2014). Encuesta La reforma universitaria desde la perspectiva de los alumnos, UAMI 2014. México D.F.: Autor (base de microdatos). 


\section{(Endnotes)}

${ }^{1}$ Encuesta que forma parte de la investigación “Los universitarios frente al cambio: reforma educativa y proyecto social. Una perspectiva regional", inscrita en los proyectos del Fondo de Investigación en Ciencia Básica SEP-CONACYT CB-201101

2 Martuscelli y Martínez (2002: 2) ubican la obra de Coombs (1971) como el motor que impulsó la idea de que las instituciones educativas atraviesan serios retos, entre ellos, "la proliferación de desequilibrios entre educación y el empleo". 